\title{
Applications of CDF in Teaching
}

\author{
Weihu Hong ${ }^{1}$, \\ ${ }^{1}$ Department of Mathematics, \\ Clayton State University, Morrow, GA, 30260,USA
}

\author{
Junfeng $Q \mathbf{u}^{2}$ \\ ${ }^{2}$ 2Department of Computer Science and Information \\ Technology, Clayton State University, Morrow, GA, \\ 30260, USA
}

\begin{abstract}
Information and Communication technologies have been rapidly developed, innovative teachings are made possible. In order to promote innovation of teaching, we will demonstrate some applications of Computable Document Format (CDF) in teaching in the form of: animation, visualization, and interaction.
\end{abstract}

Keywords: Animation, Interaction, Visualization, CDF, Active Learning

\section{Introduction}

Asinformation and communication technologies have been rapidly developed, innovation of teaching are made possible. The emerging technology makes it possible to enhance education, hands-on experience, with interactive elearning tools or technologies that support better animation, visualization and interaction between the learning contents and learner.

Edager Dale (see [5]) suggested that traditional classroom lecturing style is a passive learning style. To be more effective in passive learning, students have to be provoked and inspired. With the advances of technology, active learning that concentrates on talk, dramatic presentation, real experience, and real things is possible to be a part of learning environment in order to improve the learning quality and inspire student's interests. The cone of learning in active learning is shown in figure 1.

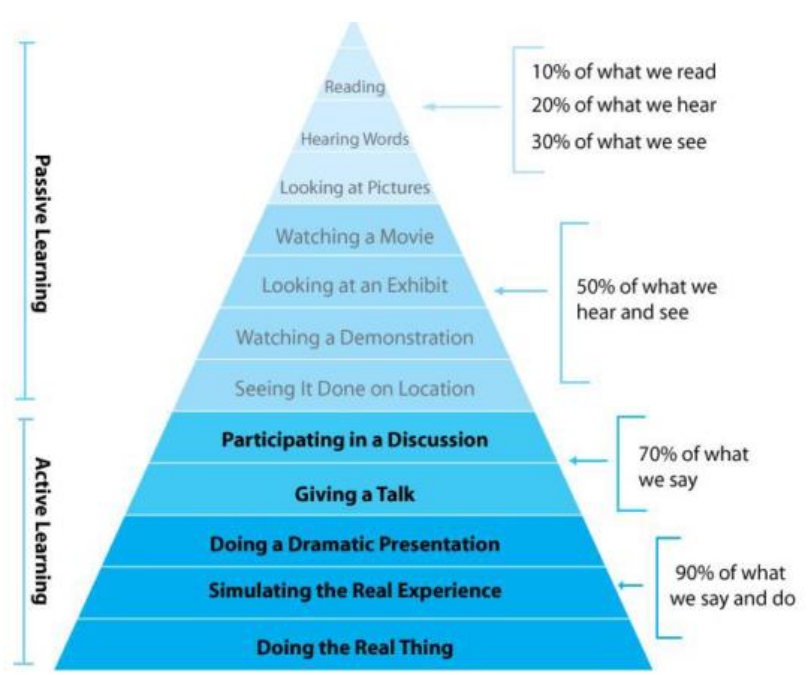

Fig. 1: The Cone of Learning

The present rich media technology, stimulates new opportunities for researchers and educators to provide and integrate video, text, audio, animation, and graphics into valuable experiences that deliver superior results for interactive teaching and learning. There have been many different explorations of applications of technology in education in the recent years such as flash, HTML5, and mathJax etc. Among all of that, the applications of Computable Document Format (CDF) from Wolfram are very significant (see [4]). The CDF offers an interactive platform for visualization, interaction, and animation, which are critical elements in active learning environments. Using the CDF, instructors can create visual depictions of data sets or models that can be later manipulated by students, and the change can be intuitively displayed on screen. If algorithms are implemented in the models or data set, the relationships among data and model variables can be shown on how these interact dynamically. The CDF contains Mathematica's core, and provides algorithms for visualization of $2 \mathrm{D}$ and $3 \mathrm{D}$. By using the computational power, the instructors could simply build interactive graphics to make the learning material more dynamic and give viewers a way to manipulate the elements by using a set of controls that would accompany the depictions. Kopecký (see [6]) presented the CDF on interactive 
learning material for teaching statistics. After study of applying the CDF on histogram, probability distribution, and then central limit theorem, the author concluded that the CDF has the potential significant effecton the development of teaching materials at all levels of education. Barry and Rachael (see [2]) studied the interactive webbased tool for learning mathematics including static and dynamic or interactive software tools. They found that the interactive resources are lacking. Web-based animations and interactive and dynamic tools are appropriate for undergraduate mathematics courses, and play an important role in mathematical education. Based on Wolfram Mathematica, the CDF serves display engine for mathematics, and makes it possible to create and deliver inline animation, interaction and visualization means with a clean and consistent interface. Although the CDF must be programmed and not supported by default by most computers, a browser plug-in or stand-along CDF player( see [3]) is free downloadable to read CDF files in the standalone desktop application or through a Web browser plug-in. It is also available for Windows, Mac OS, and Linux and mobile Oss as well. FreeCDF license is also available for non-commercial use to give users the right to use, modify and distribute CDF. Wolfram (see [10]) also host thousands of interactive documents created by Wolfram Mathematica users. In this research, we focused on applying the CDF to provide active involvement of students in learning, use the CDF as an experimental mean of understanding concept via animation, and interaction, and visualization process, and demonstrate the CDF with real-world problems that student could be able to use interactivity to further and enrich the learning experience. The examples developed in the paper are meant to be

simple (i.e. easy to use)

intuitive (no instructions necessary)

flexible (adaptable for many uses)

Aesthetic (look good and professionally designed).

\section{Animation Approach}

Animation approach is a very powerful way to help students understand concepts. Animation is not technically interactive. However, the internal relationship among variables and algorithms can be visually demonstrated and explained better with animation approach, and the concept behind can be demonstrated well. To illustrate animation with the CDF, we show how to create a demo of ten spheres system, which can be used in a classroom of studying the solar system.

Example 2.1 Ten Spheres System. If someone is teaching something about the solar system, it would be a great idea to use the animation approach. We are to show how easy it is to create an animation of a ten spheres system that will help us to deliver the information about the solar system as shown in Fig. 2.

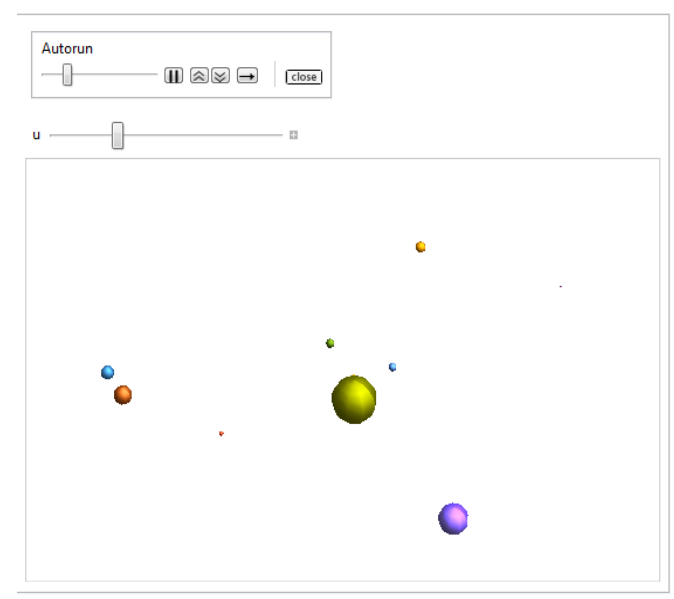

Fig. 2: Ten Spheres System

By gathering information (see [7]) such as the mean radius, the orbital period, we can write simple line of code in Mathematica (see [11]) as follows

m=Manipulate[

ParametricPlot3D [

$\{0.4 \operatorname{Cos}[\mathrm{s}] \operatorname{Sin}[\mathrm{t}]+10 \operatorname{Cos}[(2 \mathrm{Pi} / 88) \mathrm{u}]$,

$0.4 \operatorname{Sin}[\mathrm{s}] \operatorname{Sin}[\mathrm{t}]+10 \operatorname{Sin}[(2 \mathrm{Pi} / 88) \mathrm{u}]$,

$0.4 \operatorname{Cos}[\mathrm{t}]\}$,

$\{0.9 \operatorname{Cos}[\mathrm{s}] \operatorname{Sin}[\mathrm{t}]+18 \operatorname{Cos}[(2 \mathrm{Pi} / 225) \mathrm{u}]$,

$0.9 \operatorname{Sin}[\mathrm{s}] \operatorname{Sin}[\mathrm{t}]+18 \operatorname{Sin}[(2 \mathrm{Pi} / 225) \mathrm{u}]$,

$0.9 \operatorname{Cos}[\mathrm{t}]\}$,

$\{1.0 \operatorname{Cos}[\mathrm{s}] \operatorname{Sin}[\mathrm{t}]+25 \operatorname{Cos}[(2 \mathrm{Pi} / 365) \mathrm{u}]$,

1.0 $\operatorname{Sin}[\mathrm{s}] \operatorname{Sin}[\mathrm{t}]+25 \operatorname{Sin}[(2 \mathrm{Pi} / 365) \mathrm{u}]$,

$1.0 \operatorname{Cos}[\mathrm{t}]\}$,

$\{0.5 \operatorname{Cos}[\mathrm{s}] \operatorname{Sin}[\mathrm{t}]+37.5 \operatorname{Cos}[(2 \mathrm{Pi} / 687) \mathrm{u}]$,

$0.5 \mathrm{Sin}[\mathrm{s}] \mathrm{Sin}[\mathrm{t}]+37.5 \mathrm{Sin}[(2 \mathrm{Pi} / 687) \mathrm{u}]$,

$0.5 \operatorname{Cos}[\mathrm{t}]\}$,

$\{3 \operatorname{Cos}[\mathrm{s}] \operatorname{Sin}[\mathrm{t}]+40 \operatorname{Cos}[(2 \mathrm{Pi} / 4333) \mathrm{u}]$,

$3 \operatorname{Sin}[\mathrm{s}] \operatorname{Sin}[\mathrm{t}]+40 \operatorname{Sin}[(2 \mathrm{Pi} / 4333) \mathrm{u}]$,

$3 \operatorname{Cos}[t]\}$,

$\{2 \operatorname{Cos}[\mathrm{s}] \operatorname{Sin}[\mathrm{t}]+50 \operatorname{Cos}[(2 \mathrm{Pi} / 10759) \mathrm{u}]$,

$2 \operatorname{Sin}[\mathrm{s}] \operatorname{Sin}[\mathrm{t}]+50 \operatorname{Sin}[(2 \mathrm{Pi} / 10759) \mathrm{u}]$,

$2 \operatorname{Cos}[\mathrm{t}]\}$,

$\{1.5 \operatorname{Cos}[\mathrm{s}] \operatorname{Sin}[\mathrm{t}]+55 \operatorname{Cos}[(2 \mathrm{Pi} / 30687) \mathrm{u}]$,

1.5 $\operatorname{Sin}[\mathrm{s}] \operatorname{Sin}[\mathrm{t}]+55 \operatorname{Sin}[(2 \mathrm{Pi} / 30687) \mathrm{u}]$,

$1.5 \operatorname{Cos}[\mathrm{t}]\}$,

$\{1.3 \operatorname{Cos}[\mathrm{s}] \operatorname{Sin}[\mathrm{t}]+60 \operatorname{Cos}[(2 \mathrm{Pi} / 60190) \mathrm{u}]$,

1.3 $\operatorname{Sin}[\mathrm{s}] \operatorname{Sin}[\mathrm{t}]+60 \operatorname{Sin}[(2 \mathrm{Pi} / 60190) \mathrm{u}]$,

$1.3 \operatorname{Cos}[\mathrm{t}]\}$,

$\{0.2 \operatorname{Cos}[\mathrm{s}] \operatorname{Sin}[\mathrm{t}]+65 \operatorname{Cos}[(2 \mathrm{Pi} / 90465) \mathrm{u}]$,

$0.2 \operatorname{Sin}[\mathrm{s}] \operatorname{Sin}[\mathrm{t}]+65 \operatorname{Sin}[(2 \mathrm{Pi} / 90465) \mathrm{u}]$,

$0.2 \operatorname{Cos}[\mathrm{t}]\}$,

$7\{\operatorname{Cos}[\mathrm{s}] \operatorname{Sin}[\mathrm{t}], \operatorname{Sin}[\mathrm{s}] \operatorname{Sin}[\mathrm{t}], \operatorname{Cos}[\mathrm{t}]\}\}$,

$\{\mathrm{s}, 0,2 \mathrm{Pi}\},\{\mathrm{t}, 0, \mathrm{Pi}\}$, PlotRange-> All, Boxed->False, Axes->False], $\{\mathrm{u}, 0,18908\}]$

Then we run the code in Mathematica and export the file as a CDF file. Then we can load the file to a web server and insert the file into a HTML file (see [8]). 


\section{Visualization Approach}

It is very helpful in teaching by adapting visualization approach as someone said "a picture is worth a thousand words" (see [1]). There are very practical examples that were discussed in [4]. We are to share the following examples in the hope to bring more experience to the community.

Example 3.1. When we introduced a Möbius strip to students, traditionally, we would use a paper strip. With the CDF, we can visualize it on computer. In the following, we used Mathematica to create a strip in CDF as shown in Fig. 3 by using the formula

$$
\begin{aligned}
& \operatorname{MStrip}(s, t)= \\
& \begin{aligned}
<\cos (t)-s \cos \left(\frac{t}{2}\right) \cos (t), \\
\sin (t)-s \cos \left(\frac{t}{2}\right) \sin (t), \\
s \sin \left(\frac{t}{2}\right)>,
\end{aligned}
\end{aligned}
$$

for all $(s, t) \in[-0.5,0.5] \times[0,2 \pi]$

And then we write a line of code

Show[

ParametricPlot3D[

MStrip $[\mathrm{t}, \mathrm{s}] / /$ Evaluate,

$\{\mathrm{t}, 0,2 \mathrm{Pi}\},\{\mathrm{s},-0.5,-0.45\}$,

DisplayFunction $\rightarrow$ Identity,

PlotStyle $\rightarrow\{$ Yellow,Specularity[White,10]\},

PlotPoints $\rightarrow\{100,100\}$,

PlotRange $\rightarrow$ Full,Mesh $\rightarrow$ None],

ParametricPlot3D[

MStrip $[\mathrm{t}, \mathrm{s}] / /$ Evaluate, $\{\mathrm{t}, 0,2 \mathrm{Pi}\},\{\mathrm{s},-0.45,0.45\}$,

DisplayFunction $\rightarrow$ Identity,

PlotStyle $\rightarrow\{$ Blue,Specularity[White, 10] $\}$,

PlotPoints $\rightarrow\{100,100\}$,

PlotRange $\rightarrow$ Full,Mesh $\rightarrow$ None],

ParametricPlot3D[

MStrip $[\mathrm{t}, \mathrm{s}] / /$ Evaluate, $\{\mathrm{t}, 0,2 \mathrm{Pi}\},\{\mathrm{s}, 0.45,0.5\}$,

DisplayFunction $\rightarrow$ Identity,

PlotStyle $\rightarrow\{$ Yellow,Specularity[White,10]\},

PlotPoints $\rightarrow\{100,100\}$,

PlotRange $\rightarrow$ Full,Mesh $\rightarrow$ None],

DisplayFunction $\rightarrow$ \$DisplayFunction,

Axes $\rightarrow$ None,Boxed $\rightarrow$ False,PlotRange $\rightarrow$ All $]$

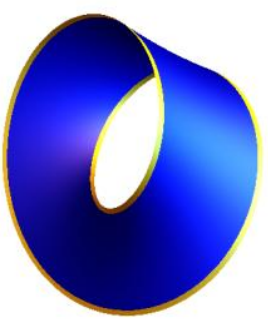

Fig. 3: Möbius Strip

Then we would ask students think of the following question: if you cut the strip along the red curve as shown in Fig. 4 then how many strips would you get?

Students would come up with different answers of course. After enough discussion, we would show a visualization after cutting, as shown in Fig. 5. The answer is one whole strip.If we ask students to think of the following question: if you cut the strip again along the middle line, then how many strips would you get? After enough discussion, we would show another visualization, as shown in Fig. 6. The answer is two strips that are tangled with each other.The above process is saved into a CDF file and deployed into a web page (see [9]).

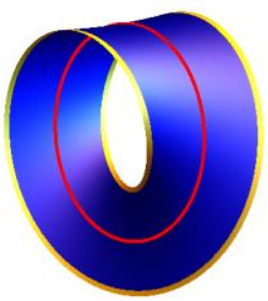

Fig. 4: Cut the strip along the red curve

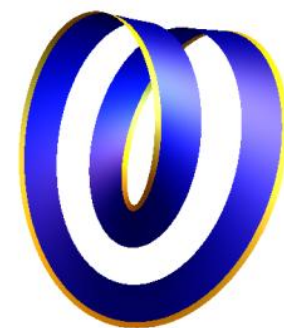

Fig. 5: One strip after the first cut 


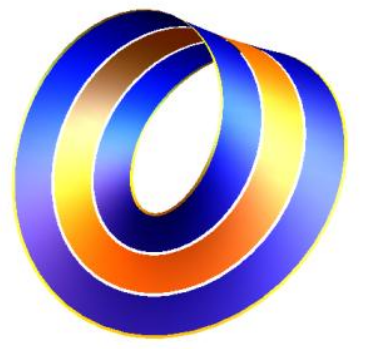

Fig. 6: Two strips after the second cut

Our next example is to visualize a topological mapping between the xy-plane and a punched sphere.

Example 3.2 Visualize the process of mapping a 2dimentional curve onto a sphere. In this example, we choose the famous Euler's spiral as the curve:

$$
\gamma(t)=<\int_{0}^{t} \sin \left(\frac{s^{2}}{2}\right) d s, \int_{0}^{t} \cos \left(\frac{s^{2}}{2}\right) d s>
$$

The spiral is shown in Fig.7.

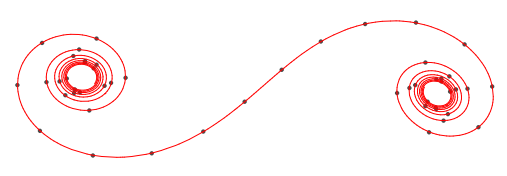

Fig. 7: Euler's spiral

Topologically speaking, the plane $\mathbb{R}^{2}$ and a punched sphere are the same. In order to map a figure in $\mathbb{R}^{2}$ onto a punched sphere, we define a mapping from the XY-plane onto the punched unit sphere with the punched point at the North Pole $(0,0,1)$ as follows

$$
\begin{aligned}
& \Gamma: X Y-\text { plane } \rightarrow S^{2} \backslash\{(0,0,1)\} \text { by } \\
& \Gamma(x, y)=\frac{2}{x^{2}+y^{2}+1}<x, y,-1>+<0,0,1>
\end{aligned}
$$

as shown in Fig. 8.

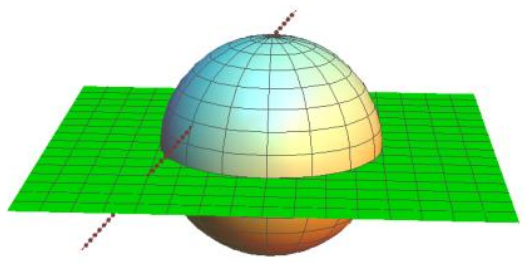

Fig. 8: The mapping between the plane and the punched sphere

If we write the curve as

$$
\gamma(\boldsymbol{t})=<x(t), \boldsymbol{y}(\boldsymbol{t}), \mathbf{0}>,
$$

its image on the sphere can be described by

$$
\Gamma(x, y)=\frac{2}{\|\gamma(t)\|^{2}+1}(\gamma(t)-k)+k
$$

Where $\boldsymbol{k}=<0,0,1>$.

It is very easy to implement the mapping using Mathematica and save it in a CDF. It results in the plot of the Euler's spiral on the sphere, as shown in Fig. 9.We can create a sphere with multiple of Euler's spirals, as shown in Fig. 10.The visualization of the mapping can be found at our website (see [9]).

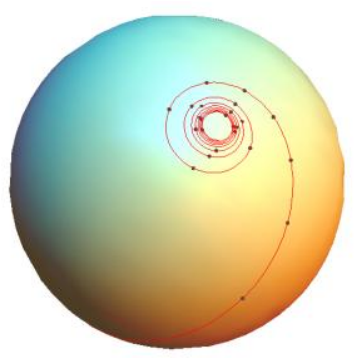

Fig. 9: Euler's spiral on the sphere

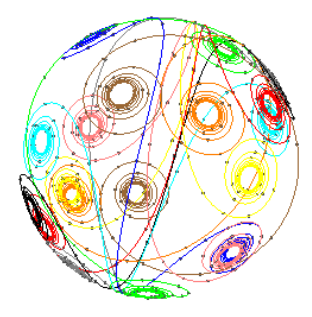

Fig. 10: Sphere with multiple of Euler's spirals. 


\section{Interactive Approach}

Interactive approach enhance animation with the capability to accept user's input and change the visualization correspondingly. Interactive graphics focus on natural senses and help viewers to develop analytical thinking in the way form single perception and experience to general knowledge (see [6]). This approach would allow users to enter different data for some parameters and observe the changes. In the following example, we demonstrate the object of hyperboloid by using the CDF.

Example 4.1 In Analytic Geometry, we deal with the object of hyperboloid that can be expressed as $\frac{x^{2}}{a^{2}}+\frac{y^{2}}{b^{2}}-$ $\frac{z^{2}}{c^{2}}=1$. It can be parametrized as

$$
<a \cos s \cosh t, b \sin s \cosh t, c \sinh t>
$$

To help students understand better about the object, we can create the object by writing the following line of code in Mathematica.

$m=$ Manipulate[ParametricPlot3D[

$\{a \operatorname{Cos}[s] \operatorname{Cosh}[t], b \operatorname{Sin}[s] \operatorname{Cosh}[t], c \operatorname{Sinh}[t]\}$,

$\{s, 0,2 \mathrm{Pi}\},\{t,-c, c\}$, PlotRange $\rightarrow$ All,

Boxed $\rightarrow$ False, Axes $\rightarrow$ False],

$\{a, 1,2\},\{b, 1,2\},\{c, 1,6\}]$

After we run the code, we save it as a CDF file and upload it to a web server. Then we insert it into a HTML page. The object is shown in Fig. 11. User can enter different values for each of the parameters $a, b$, and $c$ to view the changes of the object (see [8]).

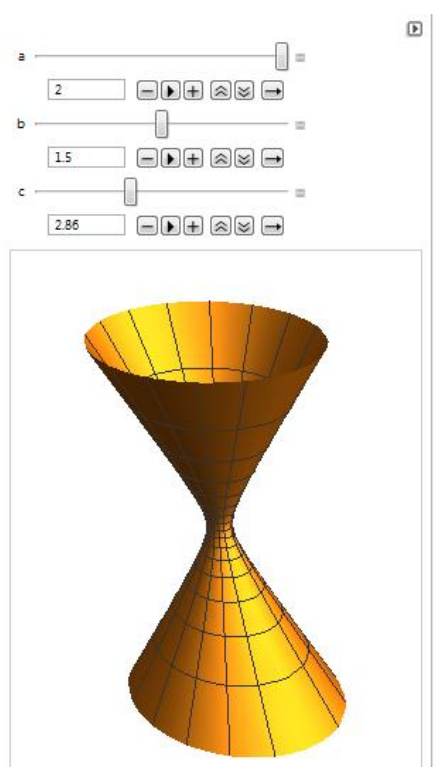

Fig. 11: Hyperboloid with $\mathrm{a}=2, \mathrm{~b}=1.5$, and $\mathrm{c}=2.86$

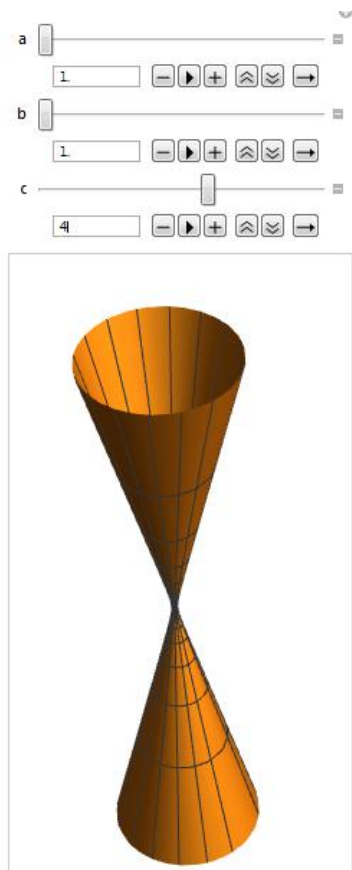

Fig. 12: Hyperboloid with $\mathrm{a}=1, \mathrm{~b}=1$, and $\mathrm{c}=4$

\section{Conclusions}

We have presented three different approaches in the applications of CDF in animation, visualization, and interaction. These three approaches are critical elements in active learning environment. CDF provides a simple and consistent mean to create rich animation, visualization, and interaction for instructors to innovate teaching. Further studies could be focus on observing the effects of such learning materials on learning and key competences of students from different fields.

\section{References}

[1] http://en.wikipedia.org/wiki/A_picture_is_worth_a_tho usand_words.

[2] Barry and Rachael "Teaching Mathematics Online: Emergent Technologies and Methodologies", IGI Global,pp.274-360, 2012)

[3] Dostupn'e z "CDF and Mathematica Features Comparison Chart". [online] http://www.wolfram.com/cdf/adopting-cdf/cdf-andmathematica-comparison.en.html.

[4] "Computable document format (cdf) for interactive content", (last viewed 22 Jan. 2013), URL https://www.wolfram.com/cdf/

[5] Dale, Edgar. Audio-Visual Methods in Teaching, 3rd ed., Holt,

Rinehart \& Winston, New York, 1969, p. 108 
[6] Kopecký, Jiři. "Using computable document format in Teaching

Mathematics." R\&E-SOURCE (2014).

[7] http://en.wikipedia.org/wiki/Solar_System

[8] http://www.gomathgo.com/csu/Animation.htm

[9] http://www.gomathgo.com/MathCAD/Main.htm

[10] http://demonstrations.wolfram.com

[11] Wolfram Research, Inc., Mathematica, 10.0 edition (Wolfram Research., Champaign, IL) (2014). 